\title{
ASSESSMENT OF WOUND HEALING ACTIVITY OF DAPHNE OLEOIDES SUBSP. KURDICA: IDENTIFICATION OF THE ACTIVE COMPONENTS USING BIOASSAY GUIDED ISOLATION METHOD
}

\author{
(๖) Küpeli Akkol Esra ${ }^{1}$, Süntar Ipek1, KelesHikmet ${ }^{2}$, Yesilada Erdem ${ }^{3}$, \\ Sarker Satyajit D. ${ }^{4}$,Arroo Randolph ${ }^{5}$, Baykal Turhan ${ }^{1}$
}

\begin{abstract}
'Department of Pharmacognosy, Faculty of Pharmacy, Gazi University, Etiler 06330, Ankara, Turkey ${ }^{2}$ Department of Pathology, Faculty of Veterinary Medicine, Afyon Kocatepe University, 03200, Afyonkarahisar, Turkey ${ }^{3}$ Department of Pharmacognosy, Faculty of Pharmacy, Yeditepe University, Atasehir 34755, Istanbul, Turkey ${ }^{4}$ Department of Pharmacy, School of Applied Sciences, University of Wolverhampton, MA Building, Wulfurna Street, Wolverhampton WV1 1LY, United Kingdom

${ }^{5}$ Leicester School of Pharmacy, De Montfort University, The Gateway, Leicester LE1 9BH, United Kingdom
\end{abstract}

In Turkish traditional medicine, the aerial parts of Daphne oleoides Schreber subsp. kurdica (DOK) have been used to treat malaria, rheumatism and for wound healing. The aim was to evaluate the ethnopharmacological usage of the plant using in vivo and in vitro pharmacological experimental models, and to perform bioassay-guided fractionation of the $85 \%$ methanolic extract of DOK for the isolation and identification of active wound-healing component (s) and to elucidate possible mechanism of the wound-healing activity. In vivo wound-healing activity was evaluated by the linear incision and the circular excision wound models. Anti-inflammatory and antioxidant activities, which are known to support the wound healing process, were also assessed by the Whittle method and the DPPH (2,2-diphenyl-1-picrylhydrazyl) radical-scavenging assays, respectively. The total phenolic content of the extract and subextracts was estimated to establish any correlation between the phenolic content and the antioxidant activity. The methanolic extract of DOK was subjected to various chromatographic separation techniques leading to the isolation and identification of the active component (s). Furthermore, in vitro hyaluronidase, collagenase and elastase enzymes inhibitory activity assays were conducted on the active components to explore the activity pathways of the remedy. After confirmation of the wound-healing activity, the methanolic extract was subjected to successive solvent partitioning using solvents of increasing polarity creating five subextracts. Each subextract was tested on the same biological activity model and the ethyl acetate (EtOAc) subextract had the highest activity. The EtOAc subextract was subjected to further chromatographic separation for the isolation of three components. The structures of these compounds were elucidated as daphnetin, demethyldaphnoretin 7-O-glucoside and luteolin 7-O-glucoside. Further in vivo testing revealed that luteolin7-O-glucoside was shown to have $39.9 \%$ increase in tensile strength in linear incision wound model and $61.2 \%$ contraction value in circular excision wound model, which were found to be significant when compared to the reference ointment Madecassol. Luteolin 7-O-glucoside was also found to exert significant anti-inflammatory, antioxidant, anti-hyaluronidase and anti-collagenase activities. The present study explored the wound-healing potential of $D$. oleoides subsp. kurdica,. Through bioassay-guided fractionation and isolation techniques, luteolin 7-O-glucoside was determined as the main active component of the aerial parts. This compound exerts its activity through inhibition of hyaluronidase and collagenase enzymes activity as well as interfering with the inflammatory stage.

\section{GASTROPROTECTIVE ACTIVITY OF THE ALCOHOLIC ROOT EXTRACT OF POTENTILLA FULGENS WALL.}

\section{(C) Laloo Damiki, Kumar Manish, Prasad Satyendra K., Sairam K., Hemalatha S.}

Department of Pharmaceutics, Institute of Technology, Banaras Hindu University, Varanasi-221005, India,

Email: dlaloo.rs.phe@itbhu.ac.in

Potentilla fulgens Wall. (Family: Rosaceae) is a short slender herb commonly found at higher altitudes of the Western Himalayas and is used traditionally for curing gastric problems, diabetes mellitus, pyorrhea and diarrhea. The aim of the present study is to scientifically evaluate the gastroprotective effect of the al- coholic root extract of (APF) on various experimental gastric ulcer models. The gastroprotective effect of APF graded doses after acute oral toxicity study (100, 200 and $400 \mathrm{mg} / \mathrm{kg}$, po) was studied on four experimental gastric ulcer models viz. pylorus ligation $(4 \mathrm{~h}$ $\mathrm{PL})$, ethanol $(95 \% \mathrm{EtOH}, 1 \mathrm{~h})$, cold restrain stress $(2 \mathrm{~h}$ 
CRS) and aspirin (4h ASP) induced ulceration. The ulcer index and \% protection was calculated. The gastric juice collected from $4 \mathrm{~h}$ PL rats was studied for the effects on gastric volume, $\mathrm{pH}$, free acid, total acid-pepsin output, DNA gastric juice cell shedding and mucosal cell proliferation. Biochemical estimation such as histamine content, mucus content and $\mathrm{H}^{+} \mathrm{K}^{+}$ATPase inhibitory activity was also evaluated. The effect of APF on stomach histopathology was also studied. Statistical analysis was done by one way ANOVA followed by Dunnett's post hoc test for multiple comparisons against control group. The difference was considered to be significant when $P<0.05$. Oral treatment of APF (200 and $400 \mathrm{mg} / \mathrm{kg}$, p. o.) for 7 days showed significant protection against acute gastric ulcer induced by $4 \mathrm{~h} P \mathrm{PL}$ and $95 \% \mathrm{EtOH}$, but was found to have no effect on CRS and ASP-induced gastric ulceration. The gastric studies showed that treatment with APF signi cantly increased the gastric juice $\mathrm{pH}$, decrease the gastric volume and total acid-pepsin output and significantly reduced the gastric juice cell shedding. A significant decrease in histamine content and a reduction in free phosphate ions responsible for $\mathrm{H}^{+} \mathrm{K}^{+}$ATPase inhibitory activity were also observed. In conclusion, the results of the present study indicate that the gastroprotective activity of APF contributes mainly to the peripheral effects on the gastric secretion and the probable mechanism underlying behind such activity might be both due to the possible inhibition on the histaminic $\left(\mathrm{H}_{2}\right)$ receptor and $\mathrm{H}^{+} \mathrm{K}^{+}$ATPase pump.

\section{INVESTIGATION OF THREE TRITERPENE ACIDS AGAINST CANCER CELL PROLIFERATION ACTIVITY}

\section{○ Khoo Gaik Ming, Clausen Morten Rahr, Larsen Erik}

Department of Food Science, Aarhus University, Kirstinebjergvej 10, DK-5792 Aarslev, Denmark

The object of this study was to investigate the inhibitory effect of betulinic acid, oleanolic acid and ursolic acid on human colon cancer cell proliferation activity. Triterpene acids are members of the terpene family and are widely distributed in food plants. A rosehip fraction containing a mixture of betulinic acid, oleanolic acid and ursolic acid displayed inhibitory effect against the release of pro-inflammatory cytokine interleukin- 6 with the combination of oleanolic acid/betulinic acid and ursolic acid/betulinic acid displaying significant synergistic inhibitory effects (1). Our results showed that these three compounds also displayed good inhibitory effect against cancer cell proliferation activity at the concentration of $10 \mu \mathrm{g} / \mathrm{ml}$. both betulinic acid and ursolic acid displayed strong inhibition against HT-29 (88\% and $79 \%$, respectively) and SW-480 (76\% and $82 \%$, respectively), but a moderate to weak inhibition against Caco-2 (19\% and $22 \%$, respectively). Oleanolic acid displayed only moderate inhibition against Caco-2 (49\%), very weak inhibition against HT-29 (7\%) and no inhibition against SW-480. No synergistic effects were observed between combinations of the triterpene acids. We only found an additive effect in the combination of oleanolic acid/ursolic acid and only against proliferation of HT-29. Antagonistic effects between the pure compounds were observed when betulinic acid was one of the components in the combination. Although betulinic acid and ursolic acid showed good inhibitory effects against all three cancer cell lines, their combination displayed antagonistic effects and showed poor inhibition against cancer cell proliferations. However, these triterpenic acids and their combination effects should be further investigated in different in vitro or in vivo experiment to confirm their bioactivities and mechanism.

Reference: (1) Saaby L., Moesby L., Hansen E.W., Christensen S.B. (2011). Isolation of immunomodulatory triterpene acids from a standardized rose hip powder (Rosa canina L.). Phytotherapy Research, 25, 195-201.

\section{DECURSIN AND RELATED COMPOUNDS INHIBIT VASCULAR ENDOTHELIAL MIGRATION BY SPHINGOSINE KINASE INHIBITION}

\section{○ Lee Yong-Moon, Shin Kyong-Oh, Seo Cho-Hee, Kim Tack-Joong}

${ }^{1}$ College of Pharmacy, Chungbuk National University, Cheongju, South Korea

${ }^{2}$ Division of Biological Science and Technology, Yonsei University, Wonju, South Korea

The pyranocoumarin compound decursin isolated from the herb, Angelica gigas, are known to possess potent anti-inflammatory activities. However, little is known about their anti-angiogenic activity. Here, we investigated the anti-angiogenic effects of decursin by the changes of sphingolipid metabolites 